\title{
Dielectric Permittivity Calculation of Composites Based on Electrospun Barium Titanate Fibers
}

\author{
H. A. Ávila*, M. M. Reboredo, R. Parra, M. S. Castro ${ }^{* *}$ \\ Instituto de Investigaciones en Ciencia y Tecnología de Materiales (INTEMA) \\ CONICET - UNMdP \\ J. B. Justo 4302, B7608FDQ Mar del Plata, Argentina \\ *havila@fi.mdp.edu.ar, ${ }^{* *}$ mcastro@fi.mdp.edu.ar
}

\begin{abstract}
On the basis of theoretical predictions and experimental results, an empirical method using upper bound equation of the rule of mixtures (ROM) is reported to predict the dielectric permittivity of barium titanate nanofibers. In addition, composites with low volume fraction of $\mathrm{BaTiO}_{3}$ fiber layers embedded in epoxy resin were prepared and characterized. The relative permittivities of composites with perpendicular and parallel configurations, with respect to the electrodes, were calculated by means of the ROM model. The predicted permittivities matched precisely the obtained experimental values.
\end{abstract}

Keywords: Polymer-matrix composites (PMCs) (A), Ceramic fiber (A), Electrical properties (B), Analytical modeling (C). 


\section{Introduction}

Composites have been extensively studied for functional and structural applications. By integrating the advantages of two phases, composite materials can offer enhanced performances with respect to those of the individual components [1]. In the last decades, there has been a great interest in polymer matrix composites that combine polymers with ferroelectric ceramics of high dielectric permittivity. Generally, the performances of this kind of composite materials depend both on the properties of the matrix and on the filler characteristics, such as its nature, geometry, orientation and volume ratio [2] besides the interphase between them. The size and concentration of ceramic particles have a significant effect on the dielectric properties of composites. Indeed, when the amount of $\mathrm{BaTiO}_{3}$ particles reaches $75 \mathrm{vol} \%$, the dielectric permittivity of nanocomposites may be increased [3]. However, defects such as voids and porosity tend to decrease the dielectric constant.The porosity of composites containing $\mathrm{BaTiO}_{3}$ microparticles decreases with the ceramic ratio up to $30 \%$, then increases abruptly at $40 \%$. The high porosity of the $40 \% \mathrm{BaTiO}_{3} /$ epoxy bulk composites leads to higher losses [4,5].

Several dielectric composites have been proposed by using $\mathrm{BaTiO}_{3}$ particles as filler and a thermoplastic [5-11] or thermosetting [12,18] polymer as matrix. Composites can be prepared in many different ways, leading to a large variety of morphologies, which strongly influence the macroscopic behavior [19]. Recently, new polymer composites using electrospun $\mathrm{BaTiO}_{3}$ fibers have been reported with promising results [20-24]. Ceramic fibers are attractive because of the increased anisotropy, improved flexibility and strength over monolithic ceramics. For instance, the potential of nanostructured composites that include $\mathrm{BaTiO}_{3}$ fibers in their compositions has been demonstrated by applications in miniaturized electronic devices and sensors [21,23,25]. Concerning different device configuration 
possibilities, a significant increase in the composite dielectric permittivity was obtained depending on the orientation of fibers inside the matrix [24].

The prediction and measurement of the dielectric properties of composites have been a challenging issue [19,26-28]. Several quantitative rules of mixture models have been proposed to calculate the dielectric permittivity of heterogeneous two-component systems considering the dielectric properties of each component [29]. However, whereas different models have been developed, usually little experimental evidence is provided to support the derived equations. Therefore, it is difficult to choose the correct model to calculate the effective dielectric permittivity of polymer/ceramic composites.

Several models have been proposed and used to predict the effects of second phases on the dielectric properties of composites such as the Maxwell-Garnett's equation [30], the Lichtenecker's equation [31], the Bruggeman's equation [32], the Jayasundere-Smith's equation [33], the Effective Medium Theory (EMT) [31] and the Poon-Shin's equation [34]. All these models take into account a continuous medium filled with spherical particles. However, only a few theoretical models are useful to predict the dielectric permittivity of polymer/ceramic-fiber composites [19].

This work aims to the estimation of the dielectric permittivity of both $\mathrm{BaTiO}_{3}$ fibers and Epoxy/BaTiO${ }_{3}$-fibers composites. The dielectric permittivity of $\mathrm{BaTiO}_{3}$ fibers has been calculated considering a fiber air-composite. Then, composite materials made of $\mathrm{BaTiO}_{3}$ fibers embedded in an epoxy resin matrix were studied. The experimental dielectric values of Epoxy/BaTiO 3 -fibers composites were matched with the ones calculated from the rule of mixtures (ROM) model. 


\section{Experimental procedure}

\section{Fiber synthesis}

For the synthesis of barium titanate fibers, a sol of titanium isopropoxide (Aldrich, 97\%), acetylacetone (Aldrich, 99.9\%) and absolute ethanol were mixed, under vigorous stirring inside a glove box, with a solution of barium acetate (Mallinckrodt) in acetic acid (Merk). A solution of poly(vinyl pyrrolidone) (PVP, 1.3MDa, Aldrich) in absolute ethanol was added to the resulting sol precursor, which was loaded into a polypropylene syringe for electrospinning. A DC voltage of $13 \mathrm{kV}$ was applied to the metallic needle by means of a Gamma High Voltage Research $(0-30 \mathrm{kV})$ unit, and a syringe pump (AcTIVA Prestigeequipment) fed the sol at a constant rate of $0.5 \mathrm{ml} / \mathrm{h}$. Random fiber mats were collected $10 \mathrm{~cm}$ below the needle tip on aluminum foil. Crystalline $\mathrm{BaTiO}_{3}$ fibers were obtained after a heat treatment in air at $800{ }^{\circ} \mathrm{C}$ for $1 \mathrm{~h}$.

\section{Structural and Microstructural Characterization of Fibers}

X-ray diffraction (XRD) was performed on fiber samples using a PANalytical, X'pert Pro equipment running with $\mathrm{CuK} \alpha$ radiation and step size of $0.02^{\circ} / \mathrm{min}$, from $10^{\circ}$ to $80^{\circ} 2 \theta$. Raman spectra were collected with a Renishaw inVia Raman microscope using a 514 nm Arion laser (50 $\mathrm{mW}$ nominal power). The microstructure and morphology of fibers were also characterized by Field Emission - Scanning Electron Microscopy (FE-SEM, Zeiss Supra 35).

\section{Preparation of Epoxy/BaTiO ${ }_{3}$ Composites}

A polymer-ceramic composite was prepared from bisphenol A-type epoxy resin (D.E.R. 331) and D.E.H. 24 (12.5\% w/w) curing agent, both from Dow Chemical, with Epodil 747 $(10 \% \mathrm{w} / \mathrm{w})$ as reactive diluent and tetrahydrofuran (THF, Dorwil Chemical) (10vol\%.) as 
solvent. Heat-treated fiber mats, in which fibers are not oriented but distributed at random, were embedded in epoxy resin inside silicone molds. The resulting composite samples were cured in an oven at $100{ }^{\circ} \mathrm{C}$ for $2 \mathrm{~h}$ for solvent removing and resin polymerization. The obtained composite was cut into specimens with dimensions in thickness, width and length, of 1,1 and $10 \mathrm{~mm}$, respectively.

\section{Dielectric Characterization of Fibers and Composites}

\subsection{Fibers}

In order to determine the effective permittivity of barium titanate fibers, the heat-treated fiber mats were considered as a biphasic composite material made of $\mathrm{BaTiO}_{3}$ and air. For this purpose, 2 and 4 layers of fiber mats were slightly pressed in order to obtain two ceramic/air composite samples with different volume fraction of ceramic filler $\left(\mathrm{V}_{\mathrm{f}}\right)$. In this way the composite material can be considered as a capacitor containing two phases that can be approximated to a structure of parallel capacitors with respect to electrodes (Figure 1)

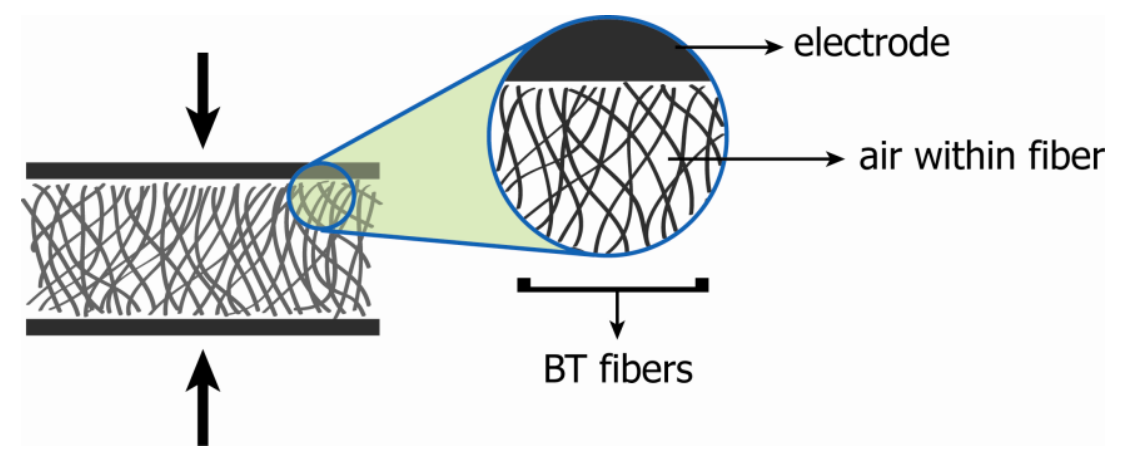

Figure 1. Schematic representation of $\mathrm{BaTiO}_{3}$ fiber layers/air composite.

\subsection{Composites}

For the dielectric characterization of composites, silver electrodes were painted on different faces of the composite samples (dimensions 1x1x10 mm), in order to obtain two 
different configurations of electrodes (parallel or perpendicular) regarding to the fiber mat layers, as shown in Figure 2, (as already described in Ávila et al. [24]).
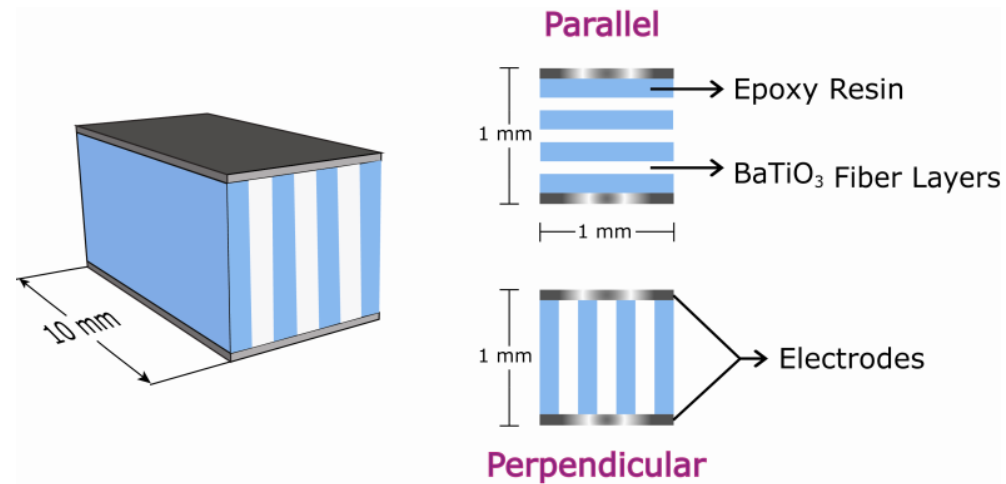

Figure 2. Epoxy/BaTiO ${ }_{3}$ fibers composites in parallel and perpendicular configurations.

In order to predict the dielectric properties of the Epoxy/BaTiO 3 composites, equation (1) was used as follows,

where, $n$ equals -1 for a parallel configuration, and +1 for a perpendicular configuration [35].

Dielectric measurements were carried out at room temperature in the $0.1-10^{7} \mathrm{~Hz}$ frequency range using HIOKI 3522-50 LCR HiTester $(10 \mathrm{mHz}-100 \mathrm{kHz})$ and HIOKI 970010 Head Amp Unit $(100 \mathrm{kHz}-120 \mathrm{MHz})$ instruments.

\section{Results and Discussion}

Figure 3(a) shows the X-ray diffraction pattern of $\mathrm{BaTiO}_{3}$ fibers heat-treated at $800{ }^{\circ} \mathrm{C}$ along with the peaks of the $\mathrm{BaTiO}_{3}$ tetragonal phase from the reference file JCPDS 74-1956. The diffraction pattern corresponds to barium titanate without secondary phases in agreement 
with the reference file. The Raman spectrum in Figure 3(b) shows typical bands for the tetragonal phase. These results demonstrate that $\mathrm{BaTiO}_{3}$ fibers consist of a mixture of cubic and tetragonal phases. The effect of thermal treatment on the crystalline structure of $\mathrm{BaTiO}_{3}$ fibers was discussed in a previous work [24].
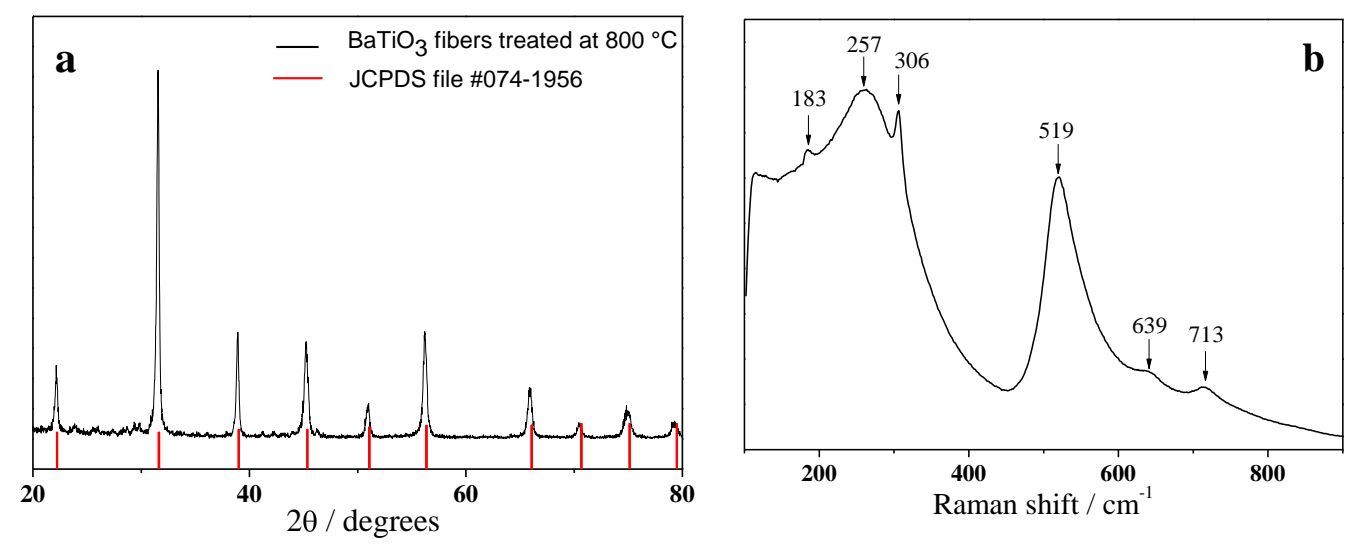

Figure 3. (a) XRD pattern and (b) Raman spectrum of $\mathrm{BaTiO}_{3}$ heat-treated fibers.

Figure 4 shows the FE-SEM images of heat-treated fibers. Figure 4(a) shows $\mathrm{BaTiO}_{3}$ fibers with average diameter of $250 \mathrm{~nm}$. The magnification in Figure 4(b) shows uniform sintered grains and mesopores generated during polymer burning. It is important to notice the continuity of fibers, which is the most important feature for determining dielectric properties of composites. 

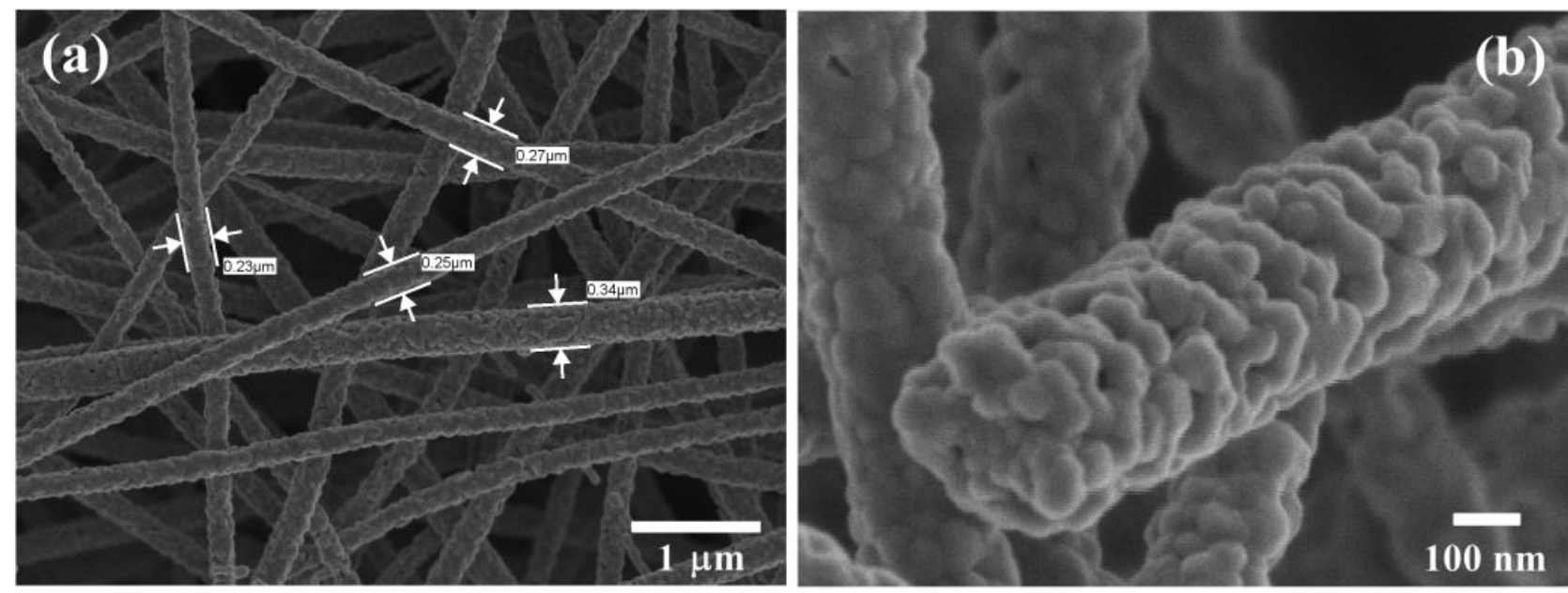

Figure 4. FE-SEM images of heat-treated electrospun $\mathrm{BaTiO}_{3}$ fibers.

Figure 5 shows a cross-section image of the Epoxy/BaTiO 3 -fiber composite fabricated with heat treated fibers. The micrograph shows dark and bright regions that belong to the epoxy resin matrix and BT fiber layers, respectively. It is clear that the epoxy resin fully covers the fibers mats and that the presence of voids or porosity in Epoxy/BT-fibers interphase is negligible.

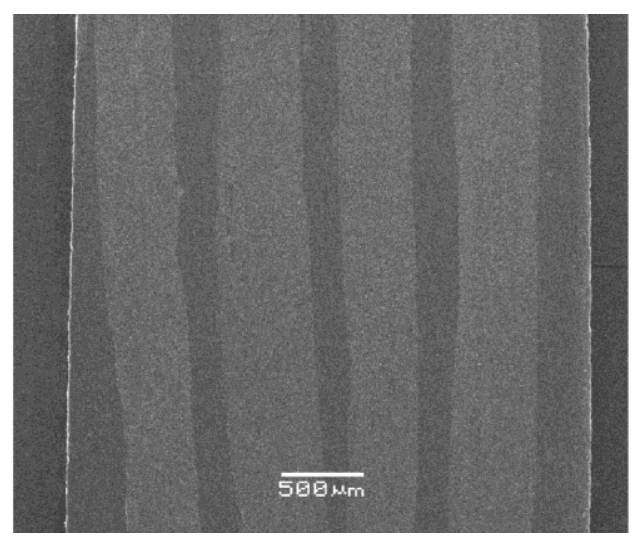

Figure 5. Cross-sectional SEM image of an Epoxy/BT-fiber composite showing fiber layers (vertical bright regions) embedded in epoxy resin (dark regions). 
The permittivity as a function of frequency for $\mathrm{BaTiO}_{3}$ fiber layers/air composites is shown in Figure 6. Taking into account the low volume fraction of fibers and the important changes measured in the permittivity values, it can be assumed that the permittivity can be approximated by a linear regression using three points with $\mathrm{V}_{f}: 0,0.0234$ and 0.157 , respectively. Then, the measured permittivity of the composite $\left(\varepsilon_{c}\right)$ can be related with the $\mathrm{BaTiO}_{3}$ (filler) permittivity $\left(\varepsilon_{f}\right)$ as follows,

where, $\varepsilon_{m}$ is the permittivity of the matrix (air relative permittivity $=1.00058$ ). Then, the permittivity of the ceramic fibers can be determined from the slope of $\varepsilon_{c} v s . V_{f}$ plots. The permittivity values of $\mathrm{BaTiO}_{3}$ fibers/air composite at specific frequency are shown in Table 1, as well as the respective linear correlation coefficients.

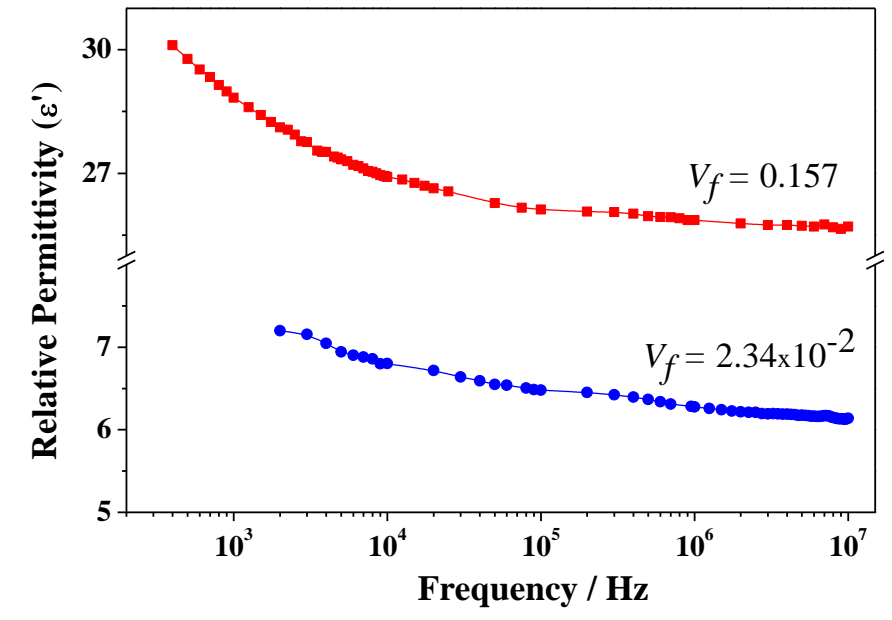

Figure 6. Relative permittivity of $\mathrm{BaTiO}_{3}$ fibers/air composites of two different volume fractions. 
Table 1. Relative permittivity values $\left(\varepsilon_{c}\right)$ of $\mathrm{BaTiO}_{3} /$ air composites with different fiber volume fractions $\left(V_{f}\right)$, calculated permittivity of fibers $\left(\varepsilon_{f}\right)$ at different frequencies using $\varepsilon_{a i r}=1.00058$, and linear correlation coefficient $\left(r^{2}\right)$.

\begin{tabular}{lcccc}
\hline & $\varepsilon_{c}$ & $\varepsilon_{c}$ & $\mathcal{E}_{f}$ & $\begin{array}{c}\text { Correlation } \\
\text { coefficient }\end{array}$ \\
\hline Frequency & $\left(\boldsymbol{V}_{f}=\mathbf{2 . 3 4 \times 1 \mathbf { 1 0 } ^ { - 2 } )}\right.$ & $\left(\boldsymbol{V}_{\boldsymbol{f}}=\mathbf{0 . 1 5 7}\right)$ & & $\boldsymbol{r}^{\mathbf{2}}$ \\
\hline $10 \mathrm{kHz}$ & 6.8 & 26.9 & 161.0 & 0.9971 \\
\hline $100 \mathrm{kHz}$ & 6.5 & 26.1 & 156.4 & 0.9974 \\
\hline $1 \mathrm{MHz}$ & 6.3 & 25.9 & 155.5 & 0.9979 \\
\hline $10 \mathrm{MHz}$ & 6.1 & 25.7 & 154.7 & 0.9983 \\
\hline
\end{tabular}

The permittivity values of $\mathrm{BaTiO}_{3}$ fibers are smaller than those of $\mathrm{BaTiO}_{3}$ powders reported by various authors as Fang et al. $\left(\varepsilon_{r}=6100\right.$ at $\left.1 \mathrm{kHz}\right)$ [36], Dutta et al. $\left(\varepsilon_{r}=500\right.$ 6000 at $1 \mathrm{kHz})$ [37], Tsurumi et al. $\left(\varepsilon_{r}=3000\right)$ [38], Ying et al. $\left(\varepsilon_{r}=800\right.$ at $\left.1 \mathrm{kHz}\right)$ [39], George et al. $\left(\varepsilon_{r}=1223\right.$ at $\left.1 \mathrm{MHz}\right)$ [40]. However, physical-morphological factors as diameter, grain size, and tetragonality could significantly affect the permittivity values of $\mathrm{BaTiO}_{3}$ fibers. Then, a direct model to predict relative permittivities, which uses few parameters and fits the experimental data, is put forward hereafter. In a recent work Wei et al. calculated the dielectric permittivity of $\mathrm{BaTiO}_{3}$ nanofibers using the Maxwell-Granett's model by considering a porous bulk specimen as a $\mathrm{BaTiO}_{3}$ fibers/air composite [41]. The high dielectric permittivity $\left(\varepsilon_{r} \sim 820\right)$ reported by the authors may be associated with the experimental procedure, by means of which fibers were pressed into pellets at $2 \mathrm{MPa}$ and heat treated at $1050^{\circ} \mathrm{C}$. It is worth noting that the applied pressure and the high temperature treatment can destroy the original fiber morphology, breaking fibers into small pieces and favoring grain growth and sintering with the consequent increase in dielectric permittivity. 
The model used in this work is simpler than that proposed by Wei et al. [41], and the experimental procedure followed does not modify or destroy the original fiber morphology.

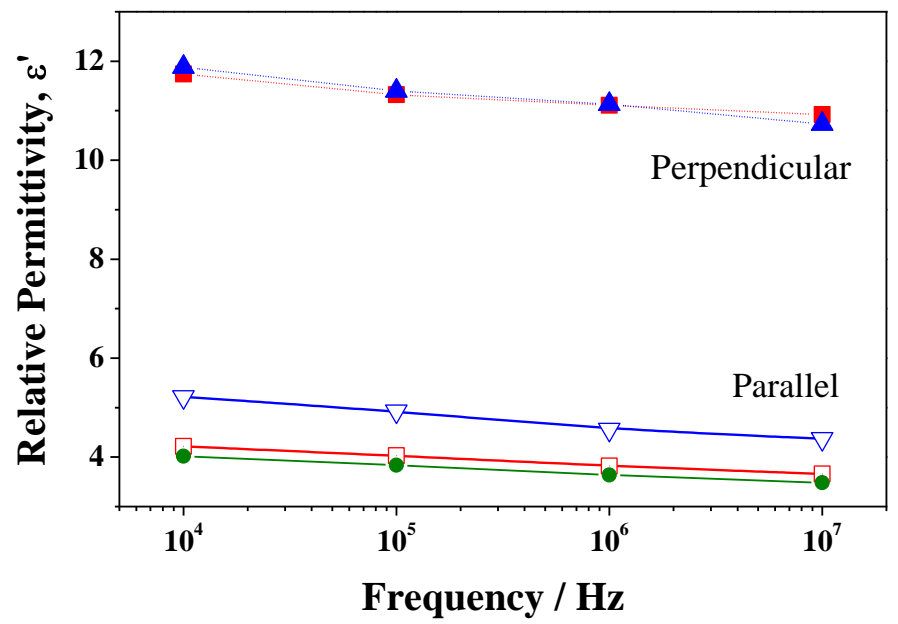

Figure 7. Permittivity of Epoxy/ $/ \mathrm{BaTiO}_{3}$ fibers composites for $4.92 \mathrm{vol} \%$ showing the experimental (blue) and calculated (red) data. The curve of the epoxy resin (green) is also shown.

In a previous work [24], a new kind of polymer/ceramic fibers composite using $\mathrm{BaTiO}_{3}$ fiber layers in parallel and perpendicular configurations with regard to the electrodes was introduced. One of the problems in predicting the effective dielectric permittivity of polymer/ceramic composites is the fact that the dielectric permittivity of the ceramic filler is not available. For biphasic composites, the highest permittivities may be calculated with equation (1) using $n=1$.

Figure 7 shows both experimental and theoretical permittivities of Epoxy/BaTiO3 fibers composites calculated using equation 2 with perpendicular $(n=1)$ and parallel $(n=-1)$ electrode configurations. The variation observed with frequency is due to the normal decay of the epoxy resin permittivity, as shown by the green curve obtained from a pure epoxy specimen. 
Some restrictions must be considered before applying the ROM model. These are: (a) the assumption that fiber layers are completely embedded in the matrix so there is perfect bonding between fibers and matrix; (b) the absence of relaxation processes in the analyzed frequency range; (c) the proposed model is only applicable for low filler fractions; (d) the absence of voids and the electrical conductivity values of filler and matrix are restricted by percolation theory.

Despite the restrictions of the ROM, the calculated values for both configurations are concomitant with experimental data. However, slight differences between experimental and theoretical permittivities, especially in the parallel arrangement, can be brought about by extrinsic defects such as air bubbles, discontinuous fibers, random orientation of fibers within the layers and residual solvent in composites.

\section{Conclusions}

A simple method has been used to determine the relative permittivity of ceramic $\left(\mathrm{BaTiO}_{3}\right)$ fibers. Then, the permittivity values obtained by means of the rule of mixtures were employed to calculate the dielectric permittivity of Epoxy/BaTiO 3 fibers composites with two different configurations. Estimations matched closely the experimental results for this kind of composites. The dielectric constant of a composite with alternating fiber-layers can be described by the rule of mixtures model equations.

\section{References}

1. Bai Y, Cheng Z-Y, Bharti V, Xu HS and Zhang QM 2000 High-dielectric-constant ceramic-powder polymer composites Appl. Phys. Lett. 76 3804-6 
2. Hammami H, Arous M, Lagache M and Kallel A 2007 Study of the interfacial MWS relaxation by dielectric spectroscopy in unidirectional PZT fibres/epoxy resin composites J. Alloy. Compd. $\mathbf{4 3 0}$ 1-8

3. Muralidhar C and Pillai PKC 1988 Dielectric behaviour of barium titanatepolyvinylidene fluoride composites J. Mater. Sci. 23 1071-6

4. Kuo D-H, Chang C-C, Su T-Y, Wang W-K and Lin B-Y 2004 Dielectric properties of three ceramic/epoxy composites Mater. Chem. Phys.85 201-6

5. Xie S-H, Zhu B-K, Wei X-Z, Xu Z-K and Xu Y-Y 2005 Polyimide/BaTiO ${ }_{3}$ composites with controllable dielectric properties Compos. Part A-Appl. S. 36 1152-7

6. Gonzalez-Benito J, Martínez-Tarifa J, Sepúlveda-García ME, Portillo RA and Gonzalez-Gaitano G 2013 Composites based on HDPE filled with $\mathrm{BaTiO}_{3}$ submicrometric particles. Morphology, structure and dielectric properties Polym. Test. 32 1342-9

7. Kim Y-H, Kim H-J, Koh J-H, Ha J-G, Yun Y-H and Nam S-M 2011 Fabrication of $\mathrm{BaTiO}_{3}-\mathrm{PTFE}$ composite film for embedded capacitor employing aerosol deposition. Ceram. Int. 37 1859-64

8. Padalia D, Bisht G, Johri U.C and Asokan K 2013 Fabrication and characterization of cerium doped barium titanate/PMMA nanocomposites Solid State Sci. 19 122-9

9. Kilic A, Shim E, Yeom BY and Pourdeyhimi B 2013 Improving electric properties of PP filaments with barium titanate $J$. Electrostat. 71 41-7

10. Wang G 2010 Enhanced dielectric properties of three-phase-percolative composites based on thermoplastic-ceramic matriz $\left(\mathrm{BaTiO}_{3}+\mathrm{PVDF}\right)$ and $\mathrm{ZnO}$ radial nanostructures ACS Appl. Mater. Interfaces 2 1290-3 
11. Choi S-H, Kim I-D, Hong J-M, Park K-H and Oh S-G 2007 Effect of the dispersibility of $\mathrm{BaTiO}_{3}$ nanoparticles in $\mathrm{BaTiO}_{3} /$ polyimide composites on the dielectric properties Mater. Lett. $612478-81$

12. Pascariu V, Padurariu L, Avadanei O and Mitoseriu L 2013 Dielectric properties of PZT-epoxy composite thick films J. Alloy. Compd. 574 591-9

13. Popielarz R, Chiang C K, Nozaki R and Obrzut J 2001 Dielectric properties of polymer/ferroelectric ceramic composites from $100 \mathrm{~Hz}$ to $10 \mathrm{GHz}$ Macromolecules 34 $5910-5$

14. Cho S-D, Lee S-Y, Hyun J-G and Paik K-W 2005 Comparison of theoretical predictions and experimental values of the dielectric constant of epoxy/BaTiO 3 composite embedded capacitor films J. Mater. Sci.-Mater. El. 16 77-84

15. Ramajo L, Castro MS and Reboredo MM 2007 Effect of silane as coupling agent on the dielectric properties of $\mathrm{BaTiO}_{3}$-epoxy composites Compos. Part A-Appl. S. 38 1852-9

16. Kuo D-H, Chang C-C, Su T-Y, Wang W-K and Lin B-Y 2001 Dielectric behaviours of multi-doped $\mathrm{BaTiO}_{3} /$ epoxy composites J. Eur. Ceram. Soc. 21 1171-7

17. Ramajo L, Reboredo MM and Castro MS 2005 Dielectric response and relaxation phenomena in composites of epoxy resin with $\mathrm{BaTiO}_{3}$ particles Compos. Part A-Appl. S. 36 1267-74

18. Zhang Z-F, Bai X-F, Zha J-W, Li W-K and Dang Z-M 2014 Preparation and dielectricp of $\mathrm{BaTiO}_{3}$ /epoxy nanocomposites for embedded capacitor application Compos. Sci. Technol. 97 100-5

19. Krakovský I and Myroshnychenko V 2002 Modeling dielectric properties of composites by finite-element method J. Appl. Phys. 92 6743- 8 
20. Ding Y, Wu Q, Zhao D, Ye W, Hanif M and Hou H 2013 Flexible $\mathrm{PI}^{-} \mathrm{BaTiO}_{3}$ dielectric nanocomposite fabricated by combining electrospinning and electrospraying Eur. Polym. J. 49 2567-71

21. Baji A, Mai Y-W, Li Q and Liu Y 2011 Nanoscale investigation of ferroelectric properties in electrospun barium titanate/polyvinylidene fluoride composite fibers using piezoresponse force microscopy Compos. Sci. Technol. 71 1435-40

22. Mimura K, Moriya M, Sakamoto W and Yogo T 2010 Synthesis of $\mathrm{BaTiO}_{3}$ nanoparticle/poly(2-hydroxyethyl methacrylate) hybrid nanofibers via electrospinning Compos. Sci. Technol. $70492-7$

23. Zhang X, Ma Y, Zhao C and Yang W 2014 High dielectric constant and low dielectric loss hybrid nanocomposites fabricated with ferroelectric polymer matrix and $\mathrm{BaTiO}_{3}$ nanofibers modified with perfluoroalkylsilane Appl. Surf. Sci. 305 531-8

24. Ávila HA, Ramajo LA, Góes MS, Reboredo MM, Castro MS and Parra R 2013 Dielectric behavior of epoxy/ $\mathrm{BaTiO}$ composites using nanostructure ceramic fibers obtained by electrospinning ACS Appl. Mater. Interfaces 5 505-10

25. Kakimoto K, Fukata $\mathrm{K}$ and Ogawa $\mathrm{H} 2013$ Fabrication of fibrous $\mathrm{BaTiO}_{3}$-reinforced PVDF composite sheet for transducer application Sensor. Actuat. A-Phys 200 21-5

26. Tuncer E, Serdyuk Y V and Gubanski S M 2002 Dielectric mixtures: electrical properties and modeling IEEE T. Dielect. El. In. 9 809-28

27. Dinulović M and Rašuo B 2011 Dielectric modeling of multiphase composites Compos. Struct. 93 3209-15

28. Hu T, Juuti J, Jantunen H and Vilkman T 2007 Dielectric properties of BST/polymer composite J. Eur. Ceram. Soc. 27 3997-4001 
29. Yamada T, Toshinobu U and Kitayama T 1982 Piezoelectricity of a high-content lead zirconate titanate/polymer composite J. Appl. Phys. 53 4328-32

30. Sihvola A 2000 Mixing rules with complex dielectric coefficients P. Soc. Photo-Opt. Ins. $1393-15$

31. Rao Y, Qu J, Marinis T and Wong C P 2000 A precise numerical prediction of effective dielectric constant for polymer-ceramic composite based on effective-medium theory IEEE Tran. Comp. Packaging Technol. 23 680-3

32. Vo H T and Shi F G 2002 Towards model-based engineering of optoelectronic packaging materials: dielectric constant modeling Microelectr. J. 33 409-15

33. Jayasundere N and Smith S B 1993 Dielectric constant for binary piezoelectric 0-3 composites J. Appl. Phys. 73 2462-6

34. Poon Y M and Shin F G 2004 A Simple explicit formula for the effective dielectric constant of binary 0-3 composites J. Mater. Sci. 39 1277-81

35. Dang Z-M, Yuan J-K, Zha J-W, Zhou T, Li S-T and Hu G-H 2012 Fundamentals, processes and applications of high-permittivity polymer-matrix composites Prog. Mater. Sci. 57 660-723

36. Fang T-T, Hsieh H-L and Shiau F-S 1993 Effects of pore morphology and grain size on the dielectric properties and tetragonal-cubic phase transition of high-purity $\mathrm{BaTiO}_{3} J$. Am. Ceram. Soc. 76 1205-11

37. Dutta P K, Asiaie R, Akbar S A and Zhu W 1994 Hydrothermal synthesis and dielectric properties of tetragonal $\mathrm{BaTiO}_{3}$ Chem. Mater. 6 1542-8

38. Tsurumi A, Sekine T, Kakemoto H, Hoshina T, Nam S-M, Yasuno H and Wada S 2006 Evaluation and statistical analysis of dielectric permittivity of $\mathrm{BaTiO}_{3}$ powders $J$. Am. Ceram. Soc. 89 1337-41 
39. Ying K-L and Hsieh T-E 2007 Sintering behaviors and dielectric properties of nanocrystalline barium titanate Mater. Sci. Eng. B 138 241-5

40. George C N, Thomas J K, Kumar H P, Suresh M K, Kumar V R, Wariar P R S, Jose R and Koshy J 2009 Characterization, sintering and dielectric properties of nanocrystalline barium titanate synthesized through a modified combustion process. Mater. Charact. 60 $322-6$

41. Wei Y, Song Y, Deng X, Han B, Zhang X, Shen Y and Lin Y 2014 Dielectric and ferroelectric properties of $\mathrm{BaTiO}_{3}$ nanofibers prepared via electrospinning J. Mater. Sci.

Technol. 30 743-7 